\title{
Axiomatizing additive multi-effort contests
}

\author{
Kjell Hausken ${ }^{1}[0$
}

Received: 28 March 2021 / Accepted: 13 September 2021 / Published online: 28 October 2021

() The Author(s) 2021

\begin{abstract}
A rent seeking model is axiomatized where players exert multiple additive efforts which are substitutable in the contest success function. The axioms assume the sufficiency of exerting one effort, and that adding an amount to one effort and subtracting the same amount from a second equivalent substitutable effort keeps the winning probabilities unchanged. In contrast, the multiplicative Cobb-Douglas production function in the earlier literature requires players to exert all their complementary efforts. The requirement follows from assuming a homogeneity axiom where an equiproportionate change in two players' matched efforts does not affect the winning probabilities. This article abandons the homogeneity axiom and assumes an alternative axiom where the winning probabilities remain unchanged when a fixed positive amount is added to all players' efforts. This article also assumes a so-called summation axiom where the winning probabilities remain unchanged when a player substitutes an amount of effort from one effort into another effort. The summation axiom excludes multiplicative production functions, and furnishes a foundation for additive production functions.
\end{abstract}

Keywords Rent seeking · Additive efforts · Axioms · Contest success function

JEL Classification C70 $\cdot$ C72 $\cdot$ D72 $\cdot$ D74

\section{Introduction}

\section{Background}

Axiomatization within the contest literature has progressed gradually. Axioms ensure solid, consistent, theoretical foundations from which other statements are logically derived. Whereas multiplicative efforts have been axiomatized in the literature, multiple additive efforts have not been axiomatized which is the objective of

Kjell Hausken

kjell.hausken@uis.no

1 Faculty of Science and Technology, University of Stavanger, 4036 Stavanger, Norway 
this article. Multiple additive efforts are relevant when contending players have multiple non-overlapping available efforts. An example is competition for elected office applying political strategists, campaigners, media operatives, speech writers, advertisements, ground troops speaking with people, etc. These efforts operate additively, not multiplicatively. The absence of one or a few efforts does not obliterate the overall effort. Rents can be procured through influence campaigns, lobbying, advertisements, product developments, fighting, etc. (Hirshleifer 1995, p. 28). Examples of rents are Research and Development budgets, licenses, privileges, election opportunities, and competition for budgets by interest groups.

\section{Contribution}

Whereas multiplicative efforts complement each other, additive efforts substitute each other in the contest success function. When multiplying the contest success function with the rent, and subtracting the expenditure of exerting efforts, a new function arises where multiple efforts can be substitutes, complements, or neither substitutes nor complements.

Influenced by Tullock (1980) and Krueger (1974), Arbatskaya and Mialon (2010, p. 24) state for multiplicative efforts that firms may obtain rents from the government by improved efficiency, or by lobbying or bribery. This presupposes that both efficiency and lobbying are required to succeed in rent seeking. Demanding that all efforts, out of many efforts, are strictly positive can be realistic for some phenomena such as career promotions to senior positions. However, for some phenomena, Cobb-Douglas type multiplicative efforts become increasingly unrealistic as the number of efforts increases since each effort must be strictly positive for success to follow.

The axiomatization of additive efforts in this article allows improved efficiency without lobbying, lobbying without improved efficiency, and, of course, both improved efficiency and lobbying operating additively. The article assumes that efforts may differ regarding unit costs, impacts, and contest intensities. Different contest intensities are not commonly analyzed in the literature.

\section{Literature}

Axiomatization is made of symmetric contests by Skaperdas (1996), asymmetric contests by Clark and Riis (1998), difference form contest success functions by Cubel and Sanchez-Pages (2016), group contests by Münster (2009), multiplicative Cobb-Douglas type efforts by Arbatskaya and Mialon (2010), contest success functions for networks by Bozbay and Vesperoni (2018), and multiple types of investments by Rai and Sarin (2009).

Blavatskyy (2010) axiomatizes contests when a draw is possible, and the highest effort does not necessarily guarantee winning. Vesperoni and Yildizparlak (2019) axiomatize single-winner contests when a draw is possible, accounting for different rents when a win or a draw occurs. Lu and Wang (2015) axiomatize multi-prize nested lottery contests. Lu and Wang (2016) axiomatize the reverse 
nested lottery contest, specifying the probabilities of being lowest ranked. Epstein and Hefeker (2003) assume that each player has two efforts; i.e. rent seeking and, if exerted, a second effort which may strengthen the first effort multiplicatively.

Yildirim (2005) assumes that two players first choose one effort each, and thereafter choose whether to add one effort. Melkonyan (2013) assumes that each player ex ante forfeits one resource and commits one resource. Ex post the winning player expends his committed resource. Hausken (2020a, b) analyzes additive efforts for multiple players. Hirshleifer (1995), Skaperdas and Syropoulos (1997) and Hausken (2005) analyze two efforts for each player labelled production and appropriation. Clark and Konrad (2007) analyze many-dimension contests, of which some have to be won to gain the rent. Keskin (2018) considers cumulative prospect theory preferences in rent seeking contests. Dickson et al. (2018) assess how rents may become more strongly contested when they become scarcer. Mercier (2018) develops a revelation mechanism where a contest designer selects a subset of contestants to maximize his profits.

Further literature considers sabotage. Konrad (2000) assumes two efforts which improves a player's contest success, and decreases the contesting players' success, respectively. Chen (2003) assumes two efforts which enhances a player's performance, and sabotages the contesting players' performance, respectively. Amegashie and Runkel (2007) analyze sabotage between four players in a threestage elimination contest. Minchuk (2021) considers reimbursement as a tool to reduce sabotaging in rent seeking contests. Krakel (2005) considers players which first help, sabotage, or abstain from action, and thereafter exert effort to win the contest. See Chowdhury and Gürtler (2015) for a survey.

Section 2 considers axioms for multiple additive efforts. Section 3 discusses the axioms and considers limitations and future research. Section 4 concludes.

\section{Axioms}

Appendix A shows the nomenclature. A static game between two players is analyzed. Player 3 is introduced as a dummy player in the axioms to facilitate the analysis, i.e. to consider subcontests between any two of three players. Player $i \in\{1,2\}$ exerts $K_{i}$ rent seeking efforts $x_{i k}, k \in\left\{1, \ldots, K_{i}\right\}$ at unit cost $c_{i k}>0$. The rent has value $S \geq 0$. Define $\boldsymbol{x}_{\boldsymbol{i}}=\left(x_{i 1}, \ldots, x_{i K_{i}}\right)$ as the vector of player $i^{\prime} s K_{i}$ efforts. Player $i^{\prime} s$ expected utility is

$$
\mathrm{u}_{i}\left(\boldsymbol{x}_{\mathbf{1}}, \boldsymbol{x}_{\mathbf{2}}\right)=p_{i}\left(\boldsymbol{x}_{\mathbf{1}}, \boldsymbol{x}_{\mathbf{2}}\right) S-\sum_{k=1}^{K_{i}} c_{i k} x_{i k},
$$

where $p_{i}^{(2)}\left(\boldsymbol{x}_{\mathbf{1}}, \boldsymbol{x}_{\mathbf{2}}\right)=p_{i}\left(\boldsymbol{x}_{\mathbf{1}}, \boldsymbol{x}_{\mathbf{2}}\right)$ and $p_{i}^{(3)}\left(\boldsymbol{x}_{\mathbf{1}}, \boldsymbol{x}_{\mathbf{2}}, \boldsymbol{x}_{3}\right)=p_{i}\left(\boldsymbol{x}_{\mathbf{1}}, \boldsymbol{x}_{\mathbf{2}}, \boldsymbol{x}_{3}\right)$ are player $i^{\prime} \mathrm{s}$ contest success functions, i.e. winning probabilities, in the two-player and threeplayer contests, respectively. For simplicity we suppress the superscripts (2) and (3) and let the number of arguments in $p_{i}$ determine the domain, i.e. $\left(\boldsymbol{x}_{\mathbf{1}}, \boldsymbol{x}_{\mathbf{2}}\right) \in \mathbb{R}_{+}^{\sum_{i=1}^{2} K_{i}}$ for the two-player contest and $\left(\boldsymbol{x}_{\mathbf{1}}, \boldsymbol{x}_{\mathbf{2}}, \boldsymbol{x}_{3}\right) \in \mathbb{R}_{+}^{\sum_{i=1}^{S} K_{i}}$ for the three-player contest. For risk neutral players, $p_{i}$ can be conceived as player $i$ 's proportion of the rent won. In 
accordance with Arbatskaya and Mialon (2010), we consider subcontests between any two of three players, $i \in I \equiv\{1,2,3\}$. We define $\boldsymbol{x}=\left(\boldsymbol{x}_{1}, \boldsymbol{x}_{\mathbf{2}}, \boldsymbol{x}_{3}\right)$ as the vector of the three players' overall number $\sum_{i=1}^{3} K_{i}$ of efforts, $x_{-i}$ as the vector of two of the players' $\sum_{j=1, j \neq i}^{3} K_{j}$ efforts except player $i^{\prime} s K_{i}$ efforts, $\boldsymbol{x}_{-i k}$ as the vector of all the three players' $\left(\sum_{i=1}^{3} K_{i}\right)-1$ efforts except player $i^{\prime} s$ effort $k \in\left\{1, \ldots, K_{i}\right\}$, and $p_{i}(\boldsymbol{x})$ as player $i^{\prime} s$ contest success function.

Axiom 1 Probability. (i) For all $i \in I$ and $x_{i k} \in \mathbb{R}_{+}^{1}$ (i.e. $x_{i k} \geq 0$ ), $p_{i}(\boldsymbol{x}) \geq 0$ and $\sum_{i \in I} p_{i}(\boldsymbol{x})=1$.

(ii) For $_{3}$ all $i \in I$, if $x_{i k} \in \mathbb{R}_{++}^{1}$ (i.e. $x_{i k}>0$ ) for at least one $k \in\left\{1, \ldots, K_{i}\right\}$, $\boldsymbol{x}_{-i k} \in \mathbb{R}_{+}^{\sum_{i=1}^{3} K_{i}-1}$, then $p_{i}(\boldsymbol{x})>0$. If $\boldsymbol{x}_{-i}=(\mathbf{0 , 0})$ and $x_{i k} \in \mathbb{R}_{++}^{1}$ for at least one $k \in\left\{1, \ldots, K_{i}\right\}$, then $p_{i}(\boldsymbol{x})=1$.

(iii) For all $i \in I$, if $\boldsymbol{x}_{\boldsymbol{i}}=\mathbf{0}, \boldsymbol{x}_{-\boldsymbol{i}} \in \mathbb{R}_{+}^{\sum_{i=1}^{2} K_{i}}$, and $\boldsymbol{x}_{-\boldsymbol{i}} \neq(\mathbf{0 , 0})$, then $p_{i}(\boldsymbol{x})=0$.

(iv) If $\boldsymbol{x}=(\mathbf{0}, \mathbf{0}, \mathbf{0})$, then $p_{1}(\boldsymbol{x})=p_{2}(\boldsymbol{x})$ and $p_{3}(\boldsymbol{x})=0$.

Axiom 1(i) assumes that a player's winning probability is non-negative regardless of all the players' efforts, and that the sum of all the players' winning probabilities is one since only one rent is allocated and the rent is inevitably allocated. Axiom 1(ii) expresses that if player $i$ exerts at least one effort which is strictly positive, then he wins with strictly positive probability, for all choices of efforts by the other players, and for all other choices of efforts by player $i$. Throughout Axiom 1 we assume that the sum of the probabilities is one. Then the whole rent $S$ always gets distributed. Arbatskaya and Mialon (2010) do not necessarily assume that all winning probabilities sum to one, in their Axiom 1(i). More specifically, they require in their Axiom (iv) that all the $K_{i}$ efforts by minimum one player must be strictly positive so that the winning probabilities sum to one. Axiom 1(ii) contrasts with Arbatskaya and Mialon (2010) who require that all player $i$ 's $K_{i}$ efforts must be strictly positive so that his probability of winning the rent is strictly positive for all possible combinations of efforts by the other players. Axiom 1(ii) also expresses that if player $i$ chooses minimally one effort which is strictly positive, while all the other players choose no efforts (i.e. efforts equal to zero), then player $i$ wins the rent with probability one. Since all the winning probabilities sum to one, all other players' winning probabilities are zero. This contrasts with Arbatskaya and Mialon (2010). Axiom 1(iii) is the same as Arbatskaya and Mialon's (2010) Axiom 1(iii), except that they do not require $\boldsymbol{x}_{-i} \neq(\mathbf{0 , 0})$ which we require to enable the new Axiom 1(iv) where all players exert no efforts. Axiom 1(iii) expresses that if player $i$ exerts no efforts, and minimally one other player exerts minimally one effort which is strictly positive, then player $i$ is guaranteed not to win the rent (i.e. wins the rent with probability zero). Axiom 1(iv) assumes that if all players withdraw from exerting effort, then the winning probabilities of players 1 and 2 are equal, while the dummy player 3's winning probably is zero.

The four parts of Axiom 1 are independent and do not imply each other for the following reasons. First, Axiom 1(i) is a simple assumption. Second, Axiom 1(ii) requires that at least one effort by player $i$ is strictly positive, i.e., at a minimum, $x_{i k} \in \mathbb{R}_{++}^{1}$. Axiom 1(ii) also assumes complementarily no effort by all the other players when $\boldsymbol{x}_{-i}=(\mathbf{0 , 0})$. Third, Axiom 1(iii) assumes no effort by player $i$, and at 
least one positive effort by at least one other player, i.e. $\boldsymbol{x}_{-i} \neq(\mathbf{0 , 0})$. Fourth, Axiom 1(iv) assumes no effort by any player, i.e. $\boldsymbol{x}=(\mathbf{0 , 0 , 0})$.

Axiom 2 Independence from irrelevant alternatives. For all $i \neq r \neq s \in I$, the odds ratio $\frac{p_{i}(\boldsymbol{x})}{p_{r}(\boldsymbol{x})}$ does not depend on $\boldsymbol{x}_{\boldsymbol{s}}$ for $\boldsymbol{x}_{\boldsymbol{i}} \in \mathbb{R}_{+}^{K_{i}}, \boldsymbol{x}_{\boldsymbol{r}} \in \mathbb{R}_{++}^{K_{r}}$, and $\boldsymbol{x}_{\boldsymbol{s}} \in \mathbb{R}_{+}^{K_{s}}$.

Axiom 2 is a version of the independence from irrelevant alternatives, and is as in Arbatskaya and Mialon (2010). It states that the ratio of any two players' winning probabilities does not depend on the third player's efforts.

Axiom 3 Inactive third player. For all $i \in\{1,2\}, \quad\left(x_{1}, x_{2}\right) \in \mathbb{R}_{+}^{\sum_{i=1}^{2} K_{i}}$, $p_{i}\left(\boldsymbol{x}_{1}, \boldsymbol{x}_{2}\right)=p_{i}\left(\boldsymbol{x}_{\mathbf{1}}, \boldsymbol{x}_{\mathbf{2}}, \boldsymbol{x}_{3}=\mathbf{0}\right)$.

Axiom 3 expresses that if the third player is inactive, then the contest reduces to a two-player contest. The number of arguments in $p_{i}$ determines the domain, as explained above. Axiom 3 is as in Arbatskaya and Mialon (2010).

Lemma 1 If Axioms 1, 2, 3 hold, then player $i$ 's contest success function, $i \in\{1,2\}$, in a two-player contest where each player $i$ exerts $K_{i}$ efforts is

$$
p_{i}\left(\boldsymbol{x}_{\mathbf{1}}, \boldsymbol{x}_{\mathbf{2}}\right)=\left\{\begin{array}{c}
\frac{f_{i}\left(\boldsymbol{x}_{i}\right)}{f_{1}\left(\boldsymbol{x}_{1}\right)+f_{2}\left(\boldsymbol{x}_{2}\right)} \text { if } x_{1 k} \in \mathbb{R}_{++}^{1} \text { for at least one } k \in\left\{1, \ldots, K_{1}\right\} \\
\quad \text { or } x_{2 k} \in \mathbb{R}_{++}^{1} \text { for at least one } k \in\left\{1, \ldots, K_{2}\right\} \\
1 / 2 \text { if } \boldsymbol{x}=(\mathbf{0}, \mathbf{0})
\end{array},\right.
$$

where player i's production (impact) function $f_{i}\left(\boldsymbol{x}_{\boldsymbol{i}}\right)$ satisfies

$$
f_{i}\left(\boldsymbol{x}_{\boldsymbol{i}}\right)\left\{\begin{array}{l}
>0 \text { if } x_{i k} \in \mathbb{R}_{++}^{1} \text { for at least one } k \in\left\{1, \ldots, K_{i}\right\} \\
\geq 0 \text { if } x_{i k} \in \mathbb{R}_{+}^{1} \text { for at least one } k \in\left\{1, \ldots, K_{i}\right\} \\
=0 \text { if } \boldsymbol{x}_{\boldsymbol{i}}=\mathbf{0}
\end{array}\right.
$$

Proof Appendix B.

Lemma 1 differs from Arbatskaya and Mialon (2010) e.g. in that it is sufficient that one of player $i^{\prime} s K_{i}$ efforts is strictly positive or positive to cause $f_{i}\left(\boldsymbol{x}_{\boldsymbol{i}}\right)$ and $p_{i}\left(\boldsymbol{x}_{\mathbf{1}}, \boldsymbol{x}_{\mathbf{2}}\right)$ to be strictly positive or positive. That implies $x_{i k} \in \mathbb{R}_{+}^{1} \backslash \mathbb{R}_{++}^{1}=\{0\}$ which is a singleton. In contrast, they demand strict positivity for all $K_{i}$ efforts by player $i$. That implies $\boldsymbol{x}_{\boldsymbol{i}} \in \mathbb{R}_{+}^{K_{i}} \backslash \mathbb{R}_{++}^{K_{i}}$ expressing a positive orthant compilation of coordinate hyperplanes. The probability $p_{i}\left(\boldsymbol{x}_{\mathbf{1}}, \boldsymbol{x}_{\mathbf{2}}\right)=0$ follows when $f_{i}\left(\boldsymbol{x}_{\boldsymbol{i}}\right)=0$ and $f_{-i}\left(\boldsymbol{x}_{-i}\right)>0$, and $p_{i}\left(\boldsymbol{x}_{\mathbf{1}}, \boldsymbol{x}_{\mathbf{2}}\right)=1$ follows when $f_{i}\left(\boldsymbol{x}_{\boldsymbol{i}}\right)>0$ and $f_{-i}\left(\boldsymbol{x}_{-i}\right)=0$.

Axiom 4 Conventional monotonicity. For all $i \neq r, i \in\{1,2\}, \quad r \in\{1,2\}$, $k \in\left\{1, \ldots, K_{i}\right\}$, and $n \in\left\{1, \ldots, K_{r}\right\}, p_{i}\left(\boldsymbol{x}_{\mathbf{1}}, \boldsymbol{x}_{\mathbf{2}}\right)$ is nondecreasing in $x_{i k}$ if $x_{i k} \in \mathbb{R}_{+}^{1}$ and $x_{r n} \in \mathbb{R}_{+}^{1}$ (i.e. $x_{i k} \geq 0$ and $x_{r n} \geq 0$ ) for at least one $n \in\left\{1, \ldots, K_{r}\right\}$, and continuous and strictly increasing in $x_{i k}$ if $x_{i k} \in \mathbb{R}_{++}^{1}$ and $x_{r n} \in \mathbb{R}_{++}^{1}$ for at least one $n \in\left\{1, \ldots, K_{r}\right\}$. 
Axiom 4 states that each player's success probability is nondecreasing in any given effort by that player if that given effort is positive and at least one effort by the other player is positive. Furthermore, each player's success probability is continuous and strictly increasing in any given effort by that player if that given effort is strictly positive and at least one effort by the other player is strictly positive. This contrasts with Arbatskaya and Mialon's (2010) Axiom 4 which requires that each player's success probability is continuous and strictly increasing with that player's efforts in each activity if the efforts by both players in every activity are positive; and otherwise, each player's success probability is nondecreasing with that player's efforts in each activity.

Axiom 5 Homogeneity. For all $i \in\{1,2\}$ and $\lambda>0, p_{i}\left(\lambda x_{1}, \lambda x_{2}\right)=p_{i}\left(x_{1}, x_{2}\right)$.

Axiom 5 equals Münster's (2009, p. 351) Axiom 6. It states that an equiproportionate change in both players' effort vectors $\boldsymbol{x}_{\mathbf{1}}$ and $\boldsymbol{x}_{\mathbf{2}}$ does not impact the players' success probabilities. This requirement differs from Arbatskaya and Mialon's (2010) Axiom 5 which requires that an equiproportionate change in corresponding single efforts $x_{1 k}$ and $x_{2 k}, k \in\left\{1, \ldots, K_{i}\right\}$, for both players does not impact the players' success probabilities. Their Axiom 5 implies Cobb-Douglas production functions. To illustrate the difference, inserting the special case $f_{i}\left(\boldsymbol{x}_{\boldsymbol{i}}\right)=x_{i 1}+x_{i 2}$ into (2) gives $p_{i}\left(\boldsymbol{x}_{\mathbf{1}}, \boldsymbol{x}_{\mathbf{2}}\right)=\frac{x_{i 1}+x_{i 2}}{x_{11}+x_{12}+x_{21}+x_{22}}($ when $\boldsymbol{x} \neq(\mathbf{0 , 0}))$. Axiom 5 is then satisfied since $\lambda$ gets abbreviated so that $p_{i}\left(\boldsymbol{x}_{\mathbf{1}}, \boldsymbol{x}_{\mathbf{2}}\right)=\frac{\lambda\left(x_{i 1}+x_{i 2}\right)}{\lambda\left(x_{11}+x_{12}\right)+\lambda\left(x_{21}+x_{22}\right)}=\frac{x_{i 1}+x_{i 2}}{x_{11}+x_{12}+x_{21}+x_{22}}$. However, if $\lambda$ is applied on only one $x_{i 1}$, and not on the whole vector $\boldsymbol{x}_{\boldsymbol{i}}$, inserting $f_{i}\left(\boldsymbol{x}_{\boldsymbol{i}}\right)=\lambda x_{i 1}+x_{i 2}$ into (2) gives $p_{i}\left(\boldsymbol{x}_{\mathbf{1}}, \boldsymbol{x}_{\mathbf{2}}\right)=\frac{\lambda x_{i 1}+x_{i 2}}{\lambda x_{11}+x_{12}+\lambda x_{21}+x_{22}}($ when $\boldsymbol{x} \neq(\mathbf{0 , 0}))$. Axiom 5 is then not satisfied since $\lambda$ cannot be abbreviated.

An alternative to homogeneity in Axiom 5 is the following where $\mathbf{1}=(1, \ldots, 1)$ is a vector with $K_{i}$ elements equal to 1 .

Axiom 6 Alternative to homogeneity. For all $i \in\{1,2\}$ and $\lambda>0$, $p_{i}\left(\boldsymbol{x}_{\mathbf{1}}+\lambda \mathbf{1}, \boldsymbol{x}_{\mathbf{2}}+\lambda \mathbf{1}\right)=p_{i}\left(\boldsymbol{x}_{\mathbf{1}}, \boldsymbol{x}_{\mathbf{2}}\right)$ if players 1 and 2 have equivalent effort technologies $f_{1}\left(\boldsymbol{x}_{\mathbf{1}}\right)=f_{2}\left(\boldsymbol{x}_{\mathbf{2}}\right)$.

Axiom 6, which departs from Arbatskaya and Mialon (2010), states that adding a constant $\lambda$ to all efforts by both players does not impact the players' success probabilities.

Axiom 7 Multiplicative and additive monotonicity. For all $i \neq s, i \in\{1,2\}$, $s \in\{1,2\}$, if $\boldsymbol{x}_{\boldsymbol{i}} b+a \mathbf{1} \geq \boldsymbol{x}_{\boldsymbol{i}}$, then $p_{i}\left(\boldsymbol{x}_{\boldsymbol{i}} b+a \mathbf{1}, \boldsymbol{x}_{\boldsymbol{s}}\right) \geq p_{i}\left(\boldsymbol{x}_{\boldsymbol{i}}, \boldsymbol{x}_{\boldsymbol{s}}\right)$.

A sufficient condition for $\boldsymbol{x}_{\boldsymbol{i}} b+a \mathbf{1} \geq \boldsymbol{x}_{\boldsymbol{i}}$ is $a \in \mathbb{R}_{+}^{1}$ and $b \geq 1$. Then Axiom 7 states that adding a positive constant $a$ to a player's effort vector $\boldsymbol{x}_{\boldsymbol{i}}$, and multiplying a player's effort vector $\boldsymbol{x}_{\boldsymbol{i}}$ with a constant $b$ larger than one, causes higher success probability. 
Axiom 8 Anonymity. For any $\left(\boldsymbol{x}_{\mathbf{1}}, \boldsymbol{x}_{\mathbf{2}}\right) \in \mathbb{R}_{+}^{\sum_{i=1}^{2} K_{i}}, p_{1}\left(\boldsymbol{x}_{\mathbf{1}}, \boldsymbol{x}_{\mathbf{2}}\right)=p_{2}\left(\boldsymbol{x}_{\mathbf{2}}, \boldsymbol{x}_{\mathbf{1}}\right)$ if $\boldsymbol{x}_{\mathbf{1}}=\boldsymbol{x}_{\mathbf{2}}$ and players 1 and 2 have equivalent effort technologies $f_{1}\left(\boldsymbol{x}_{\mathbf{1}}\right)=f_{2}\left(\boldsymbol{x}_{\mathbf{2}}\right)$.

Axiom 8 assumes that interchanging players to enable anonymity is possible only when effort technologies are equivalent.

Axiom 9 Summation. For all $i \in\{1,2\}$ and $k \in\left\{1, \ldots, K_{i}\right\}$, fixate any $\Delta_{i}>0$ such that $\Delta_{i} \leq x_{i k}$. Define

$$
\hat{\boldsymbol{x}}_{i \boldsymbol{n} \boldsymbol{n}}=\left(x_{i 1}, \ldots, x_{i, k-1}, x_{i k}-\Delta_{i}, x_{i, k+1}, \ldots, x_{i, n-1}, x_{i n}+\Delta_{i}, x_{i, n+1}, \ldots, x_{i K}\right) .
$$

Then

$$
p_{i}\left(\hat{\boldsymbol{x}}_{i k n}, \boldsymbol{x}_{-i}\right)=p_{i}\left(\boldsymbol{x}_{\boldsymbol{i}}, \boldsymbol{x}_{-i}\right)
$$

assuming that efforts $x_{i k}$ and $x_{i n}$ in the contest success function $p_{i}\left(\boldsymbol{x}_{\mathbf{1}}, \boldsymbol{x}_{\mathbf{2}}\right)$ are substitutable for all $k \in\left\{1, \ldots, K_{i}\right\}$ and $n \in\left\{1, \ldots, K_{i}\right\}$ while preserving $\Delta_{i}>0$.

Axiom 9 states that if two efforts $x_{i k}$ and $x_{i n}$ are substitutable in the contest success function $p_{i}\left(\boldsymbol{x}_{\mathbf{1}}, \boldsymbol{x}_{\mathbf{2}}\right)$, and player $i$ exerts less effort, by the amount $\Delta_{i}$, into effort $k$ yielding effort $x_{i k}-\Delta_{i}$, and exerts more effort, by the same amount $\Delta_{i}$, into effort $n$ yielding effort $x_{i n}+\Delta_{i}$, then the winning probabilities of both players are unaffected. Hence only the sum $x_{i k}-\Delta_{i}+x_{i n}+\Delta_{i}=x_{i k}+x_{i n}$ matters, independently of $\Delta_{i}$. Axiom 9 equals Münster's (2009, p. 354) Axiom 8 for groups where, if one group member exerts more effort while another group member exerts equally less effort, the group's winning probability remains unchanged.

Lemma 2 Consider any contest success function $p_{i}\left(\boldsymbol{x}_{\mathbf{1}}, \boldsymbol{x}_{\mathbf{2}}\right)$ from the class characterized in Lemma 1 . A success function $p_{i}\left(\boldsymbol{x}_{\mathbf{1}}, \boldsymbol{x}_{\mathbf{2}}\right)$ satisfying Axioms $1,2,3,4$, 7 is compatible with a CES production (impact) function $f_{i}\left(\boldsymbol{x}_{\boldsymbol{i}}\right)$ taking the form ${ }^{1}$

$$
f_{i}\left(\boldsymbol{x}_{\boldsymbol{i}}\right)=\left(\sum_{k=1}^{K_{i}} d_{i k} x_{i k}^{m_{i k}}\right)^{r},
$$

where $d_{i k} \geq 0$ scales impact proportionally, $m_{i k} \geq 0$ is player $i$ 's contest intensity, and $r \geq 0$. Axiom 5 is compatible with (6) when $m_{i k}=m$. Axiom 8 is compatible with (6) when $\boldsymbol{x}_{\mathbf{1}}=\boldsymbol{x}_{\mathbf{2}}, d_{1 k}=d_{2 k}, m_{1 k}=m_{2 k}, K_{1}=K_{2}$. Axiom 9 is compatible with (6) when $d_{i k}=d_{\text {in }}$ and $m_{i k}=m_{i n}=1, k, n \in\left\{1, \ldots, K_{i}\right\}$, required for substitutability between $x_{i k}$ and $x_{i n}$ in Axiom 9.

Proof Münster (2009, p. 355) proves Lemma 2 in his Proposition 2 assuming his axioms A1, A2, A4, A5, A6, A8 for the special case that $d_{i k}=d_{i}$ and $m_{i k}=1$. Appendix C generalizes Münster's (2009) proof from $x_{i k}$ to $d_{i k} x_{i k}^{m_{i k}}$ inside the

\footnotetext{
${ }^{1}$ Equation (6) is a generalization of the CES (Constant elasticity of substitution) function common in production economics.
} 
summation applying Axioms 1, 2, 3, 4, 7, some of which correspond to Münster's (2009) axioms.

Lemma 3 Consider any contest success function $p_{i}\left(\boldsymbol{x}_{\mathbf{1}}, \boldsymbol{x}_{\mathbf{2}}\right)$ from the class characterized in Lemma 1 . A success function $p_{i}\left(\boldsymbol{x}_{\mathbf{1}}, \boldsymbol{x}_{\mathbf{2}}\right)$ satisfying Axioms $1,2,3,4,7$ is compatible with a production (impact) function $f_{i}\left(\boldsymbol{x}_{\boldsymbol{i}}\right)$ taking the logistic form

$$
f_{i}\left(\boldsymbol{x}_{\boldsymbol{i}}\right)=a_{i} \operatorname{Exp}\left(r \sum_{k=1}^{K_{i}} d_{i k} x_{i k}^{m_{i k}}\right),
$$

where $d_{i k} \geq 0, m_{i k} \geq 0, a_{i} \geq 0$, and $r \geq 0$. Axiom 6 is compatible with (7) when $d_{1 k}=d_{2 k}, m_{i k}=1, K_{1}=K_{2}$.

Proof Münster (2009, pp. 355-356) proves Lemma 3 in his Proposition 3 by contradiction assuming his axioms A1, A2, A4, A5, A7, A8 for the special case that $d_{i k}=d_{i}$ and $m_{i k}=1$. Appendix D generalizes Münster's (2009) proof from $x_{i k}$ to $d_{i k} x_{i k}^{m_{i k}}$ inside the summation applying Axioms 1, 2, 3, 4, 7, some of which correspond to Münster's (2009) axioms.

Whereas $p_{i}\left(\boldsymbol{x}_{\mathbf{1}}, \boldsymbol{x}_{\mathbf{2}}\right)$ interpreted as the ratio form contest success function (Skaperdas, 1996; Tullock, 1980) in Lemma 2 is compatible with Axiom 5 when $m_{i k}=m$, $p_{i}\left(\boldsymbol{x}_{\mathbf{1}}, \boldsymbol{x}_{\mathbf{2}}\right)$ interpreted as the logistic contest success function (Hirshleifer, 1989) in Lemma 3 is compatible with Axiom 6 when $d_{1 k}=d_{2 k}, m_{i k}=1, K_{1}=K_{2}$. Arbatskaya and Mialon (2010) also assume contest intensities $m_{i k}=1$. A contest success function $p_{i}\left(\boldsymbol{x}_{\mathbf{1}}, \boldsymbol{x}_{\mathbf{2}}\right)$ and production (impact) function $f_{i}\left(\boldsymbol{x}_{\boldsymbol{i}}\right)$ from the classes characterized in Lemma 2 is compatible with Axiom 9 only for the special case that $d_{i k}=d_{i n}$ and $m_{i k}=m_{i n}=1, k, n \in\left\{1, \ldots, K_{i}\right\}$. That special case, usually restricted to $d_{i k}=d_{i n}=1$, is the case commonly studied in the literature, and thus constitutes a comparison benchmark.

Lemma 4 Consider any contest success function $p_{i}\left(\boldsymbol{x}_{\mathbf{1}}, \boldsymbol{x}_{\mathbf{2}}\right)$ from the class characterized in Lemma 1. If this success function $p_{i}\left(\boldsymbol{x}_{\mathbf{1}}, \boldsymbol{x}_{\mathbf{2}}\right)$ satisfies Axiom 1(ii), or Axiom 9, its production (impact) function $f_{i}\left(\boldsymbol{x}_{\boldsymbol{i}}\right)$ cannot take the form of the Cobb-Douglas production function

$$
f_{i}\left(\boldsymbol{x}_{\boldsymbol{i}}\right)=d_{i} \prod_{k=1}^{K_{i}} x_{i k}^{m_{i k}} .
$$

In contrast, if $p_{i}\left(\boldsymbol{x}_{\mathbf{1}}, \boldsymbol{x}_{\mathbf{2}}\right)$ in Lemma 1 satisfies Axiom 5 and $m_{i k}=m$, it is possible for $f_{i}\left(\boldsymbol{x}_{\boldsymbol{i}}\right)$ to take the form in (8).

Proof Appendix E.

Axiom 9 drives a wedge between the additive production function in (6) and multiplicative production functions. Axiom 9 is compatible with (6) when $d_{i k}=d_{i n}$ 
and $m_{i k}=m_{i n}=1, k, n \in\left\{1, \ldots, K_{i}\right\}$. Axiom 9 excludes multiplicative production functions including Cobb-Douglas production functions since it is mathematically impossible to find one special case where a fixed amount $\Delta_{i}$ is added to one effort and subtracted from another effort while, simultaneously, the product of the efforts, where each effort is raised to an exponent as in the Cobb-Douglas production function, remains unchanged. The model has $3 K_{1}+3 K_{2}+2$ parameters, i.e. $K_{1}+K_{2}$ unit efforts costs $c_{i k}, K_{1}+K_{2}$ scaling parameters $d_{i k}, K_{1}+K_{2}$ contest intensities $m_{i k}$, and $K_{1}+K_{2}$ for the numbers of efforts $x_{i k}$ which are the players' strategic choice variables.

\section{Discussion, limitations and future research}

This article's departs from Arbatskaya and Mialon (2010) is Axiom 6 which states that winning probabilities remain unchanged when a fixed positive amount is added to all players' efforts. One advantage of Axiom 6 is that it allows for additive efforts and therefore efforts can be perfect substitutes, in contrast to Arbatskaya and Mialon (2010). That efforts might be perfect substitutes affects the distribution of efforts among different activities which has important consequences when analyzing possible equilibria, rent dissipation, contest design, etc., as illustrated by Hausken (2020a, b).

Axiom 6 is reminiscent of Translation Invariance but imposes directly a restriction on player $i$ 's production (impact) function $f_{i}\left(\boldsymbol{x}_{\boldsymbol{i}}\right)$ rather than player $i$ 's contest success function $p_{i}(\boldsymbol{x})$. Future research may develop this further to obtain a restriction on $p_{i}(\boldsymbol{x})$ which then could lead to a functional representation via the existence of some $f_{i}\left(\boldsymbol{x}_{\boldsymbol{i}}\right)$.

The functional forms in this article are restricted to a class that coincides with the one characterized in Lemma 1 of Arbatskaya and Mialon (2010) except for cases with zero efforts. These cases are covered in Axiom 1 which specifies whether a winning probability should be zero when certain efforts are zero. Axioms 1, 2 and 3 are otherwise similar to the corresponding axioms in Arbatskaya and Mialon (2010) and lead to an analogous characterization. This limits the value of Lemma 1.

Axioms 4-9 state variations of conventional properties of success functions. That is, Axiom 4 expresses standard monotonicity, and Axiom 5 expresses standard momogeneity (or Scale Invariance). Axiom 7 is a variation of Monotonicity. Axiom 8 is a weaker form of the standard Anonymity axiom. It requires symmetry only if effort vectors are symmetric across players, whereas the standard anonymity axiom is typically based on permutation of identities. Axiom 9 is a form of Independence influenced by Münster's (2009) Axiom 8. It serves the analogous purpose of identifying a linear form for $f_{i}\left(\boldsymbol{x}_{\boldsymbol{i}}\right)$. Axiom 9 crucially excludes multiplicative production functions including Cobb-Douglas production functions, and furnishes a foundation for additive production functions.

One limitation of the article is that Axioms 4-9 do not lead to any characterization, that is, a statement claiming that a contest success function satisfies a set of axioms if and only if it takes a certain functional form. Instead, Axioms 4-9 are 
employed in Lemmas 2, 3 and 4 which express compatibility between axioms and functional forms. Future research should extend these expressions on compatibility to "if and only" characterizations.

Future research should furthermore axiomatize more general contest success functions and production functions (e.g. which depend less separably on the multiple efforts), consider more than two players, and analyze empirically which forms are descriptive, including when multiple efforts are additive or multiplicative.

\section{Conclusion}

Axioms and lemmas are developed for rent seeking where two players exert multiple additive efforts. The implication for theory is that the satisfied axioms assume the sufficiency of exerting one effort under certain conditions, because efforts can substitute for each other. On the other hand, the satisfied axioms for the multiplicative Cobb-Douglas production function analyzed in the earlier literature require that all available efforts are exerted by a player, because efforts complement each other. The additive production function furthermore assumes a so-called summation axiom where adding an amount to one effort and subtracting the same amount from a second equivalent substitutable effort does not change the players' probabilities of winning the contest. In contrast, the Cobb-Douglas production function analyzed in the earlier literature assumes a homogeneity axiom where an equiproportionate change in two players' matched efforts does not affect the probabilities of winning.

The implication for practice is that an axiomatic foundation provides rent seeking practitioners, regulators, analysts and others with a more rigorous basis for understanding the role, logic, mechanism, and impact of rent seeking with multiple efforts. This article informs candidates for elected office, entrepreneurs seeking government privileges, firms seeking various rents, and policy makers, how to understand when multiple efforts by their nature are additive or multiplicative. The article specifies when multiple efforts are substitutes, complements, or neither substitutes nor complements, and how multiple efforts impact when they have different relevance, different unit costs, different impacts, and different contest intensities.

Supplementary Information The online version contains supplementary material available at https://doi. org/10.1007/s43546-021-00155-5.

Funding Open access funding provided by University of Stavanger. No funding was received.

Data availability All relevant data are included in the article.

\section{Declarations}

Conflict of interest No conflict of interest exists.

Ethical approval No animals or humans were involved. 
Open Access This article is licensed under a Creative Commons Attribution 4.0 International License, which permits use, sharing, adaptation, distribution and reproduction in any medium or format, as long as you give appropriate credit to the original author(s) and the source, provide a link to the Creative Commons licence, and indicate if changes were made. The images or other third party material in this article are included in the article's Creative Commons licence, unless indicated otherwise in a credit line to the material. If material is not included in the article's Creative Commons licence and your intended use is not permitted by statutory regulation or exceeds the permitted use, you will need to obtain permission directly from the copyright holder. To view a copy of this licence, visit http://creativecommons.org/licen ses/by/4.0/.

\section{References}

Amegashie JA, Runkel M (2007) Sabotaging potential rivals. Soc Choice Welf 28(1):143-162. https:// doi.org/10.1007/s00355-006-0157-4

Arbatskaya M, Mialon HM (2010) Multi-activity contests. Econ Theor 43(1):23-43. https://doi.org/10. 1007/s00199-008-0424-y

Blavatskyy PR (2010) Contest success function with the possibility of a draw: axiomatization. J Math Econ 46(2):267-276. https://doi.org/10.1016/j.jmateco.2009.11.012

Bozbay I, Vesperoni A (2018) A contest success function for networks. J Econ Behav Organ 150:404422. https://doi.org/10.1016/j.jebo.2018.01.022

Carter M (2001) Foundations of mathematical economics. MIT Press, Cambridge

Chen KP (2003) Sabotage in promotion tournaments. J Law Econ Organ 19(1):119-140. https://doi.org/ 10.1093/jleo/19.1.119

Chowdhury S, Gürtler O (2015) Sabotage in contests: a survey. Public Choice 164(1):135-155. https:// doi.org/10.1007/s11127-015-0264-9

Clark DJ, Konrad KA (2007) Contests with multi-tasking. Scand J Econ 109(2):303-319. https://doi.org/ 10.1111/j.1467-9442.2007.00495.x

Clark DJ, Riis C (1998) Contest success functions: an extension. Econ Theor 11(1):201-204. https://doi. org/10.1007/s001990050184

Cubel M, Sanchez-Pages S (2016) An axiomatization of difference-form contest success functions. J Econ Behav Organ 131:92-105. https://doi.org/10.1016/j.jebo.2016.08.011

Dickson A, MacKenzie IA, Sekeris PG (2018) Rent-seeking incentives in share contests. J Public Econ 166:53-62. https://doi.org/10.1016/j.jpubeco.2018.08.004

Epstein GS, Hefeker C (2003) Lobbying contests with alternative instruments. Econ Gov 4(1):81-89. https://doi.org/10.1007/s101010200049

Hausken K (2005) Production and conflict models versus rent-seeking models. Public Choice 123(12):59-93. https://doi.org/10.1007/s11127-005-1717-3

Hausken K (2020a) Additive multi-effort contests. Theor Decis 89(2):203-248

Hausken K (2020b) Additive multi-effort contests with multiple investment opportunities. Appl Econ Lett 27(1):67-71

Hirshleifer J (1989) Conflict and rent-seeking success functions-ratio vs difference models of relative success. Public Choice 63(2):101-112. https://doi.org/10.1007/bf00153394

Hirshleifer J (1995) Anarchy and its breakdown. J Polit Econ 103(1):26-52

Keskin K (2018) Cumulative prospect theory preferences in rent-seeking contests. Math Soc Sci 96:8591. https://doi.org/10.1016/j.mathsocsci.2018.06.002

Konrad KA (2000) Sabotage in rent-seeking contests. J Law Econ Organ 16(1):155-165. https://doi.org/ $10.1093 /$ jleo/16.1.155

Krakel M (2005) Helping and sabotaging in tournaments. Int Game Theory Rev 7(2):211-228

Krueger AO (1974) The political economy of the rent-seeking society. Am Econ Rev 64(3):291-303

Lu JF, Wang ZW (2015) Axiomatizing multi-prize nested lottery contests: a complete and strict ranking perspective. J Econ Behav Organ 116:127-141. https://doi.org/10.1016/j.jebo.2015.04.010

Lu JF, Wang ZW (2016) Axiomatization of reverse nested lottery contests. Soc Choice Welf 47(4):939957. https://doi.org/10.1007/s00355-016-0998-4

Melkonyan T (2013) Hybrid contests. J Public Econ Theory 15(6):968-992. https://doi.org/10.1111/jpet. 12052 
Mercier JF (2018) Selecting contestants for a rent-seeking contest: a mechanism design approach. Int J Game Theory 47(3):927-947. https://doi.org/10.1007/s00182-017-0610-x

Minchuk Y (2021) Reimbursement as a tool to reduce sabotaging in rent-seeking contests. Manag Decis Econ 42(1):235-238. https://doi.org/10.1002/mde.3228

Münster J (2009) Group contest success functions. Econ Theor 41(2):345-357. https://doi.org/10.1007/ s00199-009-0479-4

Rai B, Sarin R (2009) Generalized contest success functions. Econ Theor 40(1):139-149. https://doi.org/ 10.1007/s00199-007-0328-2

Skaperdas S (1996) Contest success functions. Econ Theor 7(2):283-290. https://doi.org/10.1007/s0019 90050053

Skaperdas S, Syropoulos C (1997) The distribution of income in the presence of appropriative activities. Economica 64(253):101-117. https://doi.org/10.1111/\%28ISSN\%291468-0335/issues

Tullock G (1980) Efficient rent-seeking. In: Buchanan JM, Tollison RD, Tullock G (eds) Toward a theory of the rent-seeking society. Texas A. \& M. University Press, College Station, pp 97-112

Vesperoni A, Yildizparlak A (2019) Contests with draws: axiomatization and equilibrium. Econ Inq 57(3):1597-1616. https://doi.org/10.1111/ecin.12780

Yildirim H (2005) Contests with multiple rounds. Games Econ Behav 51(1):213-227. https://doi.org/10. 1016/j.geb.2004.03.004 